\title{
Largest separable balls around the maximally mixed bipartite quantum state
}

\author{
Leonid Gurvits and Howard Barnum \\ CCS-3, Mail Stop B256, Los Alamos National Laboratory, Los Alamos, NM 87545
}

(Dated: October 22, 2018)

\begin{abstract}
For finite-dimensional bipartite quantum systems, we find the exact size of the largest balls, in spectral $l_{p}$ norms for $1 \leq p \leq \infty$, of separable (unentangled) matrices around the identity matrix. This implies a simple and intutively meaningful geometrical sufficient condition for separability of bipartite density matrices: that their purity $\operatorname{tr} \rho^{2}$ not be too large. Theoretical and experimental applications of these results include algorithmic problems such as computing whether or not a state is entangled, and practical ones such as obtaining information about the existence or nature of entanglement in states reached by NMR quantum computation implementations or other experimental situations.
\end{abstract}

PACS numbers: 03.65.Ud,03.67.-a,03.67.Lx

\section{INTRODUCTION AND SUMMARY OF RESULTS}

Entanglement is an important element of many quantum information processing procedures, from cryptography to computation to quantum teleportation. Indeed, a quantum algorithm operating on pure quantum states must entangle a number of qubits increasing unboundedly with the input size, if it is not to be simulable in polynomial time on a classical computer [1]. It is not known whether this is so when the computer state may be mixed. Determining whether a given state, even of two quantum systems, is entangled or separable (not entangled) is in general difficult, and considerable effort has been expended on finding necesssary and/or sufficient conditions. The normalized separable states form a convex set. A key aspect of the geometry of a convex set is the size of the largest ball (especially in $l_{2}$ norm) that fits entirely inside it, and the smallest ball that covers it. We here find the inner ball for the set of separable quantum states. The result has both practical and theoretical relevance. For example, it provides a simple sufficient criterion for separability. A bipartite state of a composite system with overall dimension $d$ is separable if its purity $\operatorname{tr} \rho^{2}$ is less than $1 /(d-1)$ (as conjectured in [2]). Because of its simple geometric nature and ease of computation, this criterion is likely to be very useful both in theoretical applications and in analyzing whether entanglement was present in experiments. Just as importantly, knowing the size of such balls helps one understand the computational complexity of problems involving a convex set. For example, using bounds on the size of the inner ball (rather than the exact result we here present) one of us has shown the NP-hardness of the "weak membership" problem for separability when the dimensions of the two systems are not too different [8]; it is likely that the exact result reported herein may be used in extending this hardness result or in obtaining other complexity results about separability and entanglement.

Our main results begin with Theorem 1, that the matrix $I+\Delta$ is separable for all hermitian $\Delta$ with $\|\Delta\|_{2} \leq 1$. Corollary 1 gives similar statements for other $p$-norms.
Theorem 3 establishes that for $2 \leq p \leq \infty$, these are the largest such balls. A similar result is easy for $1 \leq p \leq 2$; it involves subtracting a normalized pure state to reach the edge of the ball, where the state becomes singular and thus is at the edge of the positive cone. Theorem 4 tells us about the size of the largest negative eigenvalues of the partial transpose of bipartite positive matrices that are rank- $m$ projectors, giving us information about how quickly we can hit the entangled matrices when departing from the identity by adding a positive multiple of such a projector. In particular, by considering a perturbation $\Delta$ proportional to such a projector whose partial transpose has maximal modulus of its most negative eigenvalue, it can be used to show that for $p=2$ the largest $p$-norm ball around $I$ touches the edge of the separable cone at places within the positive cone.

As one example of practical relevance, the "pseudopure" states which describe each molecule in NMR quantum information processing are mixtures of the uniform density matrix with a pure state; the signal of quantum dynamics derives from the small pure component. Because of this, the density matrices of the different nuclear spins in a given molecule have not, in experiments so far done, exhibited entanglement despite the pure component being an entangled state; they have remained within known lower bounds on the size of the ball of separable states [3]. Our determination of the exact size of this ball for bipartite entanglement increases known lower bounds on the polarization necessary in order for such bulk computation on pseudopure states to be able to achieve bipartite entanglement, although due to the bipartite nature of our analysis, it does not rule out the production of entangled states that are separable with respect to every bipartition, at lower polarization. This raises the interesting question whether the exponential gap between our bound for bipartite separability and known bounds for separability in this context, can be closed. Other quantum information processing procedures may also involve such mixtures; also, mixture with the identity matrix is a frequently studied model of quantum noise, the "depolarizing channel," to which our results are relevant. When this occurs in bipartite contexts, our results are 
much stronger than previously known. We emphasize, though, that the sufficient conditions for entanglement and separability provided by our results apply in arbitrary contexts, not only for mixtures of a pure state with the normalized identity.

\section{MATHEMATICAL BACKGROUND AND NOTATION}

We represent unnormalized states of a quantum system composed of two subsystems of dimensions $M$ and $N$ (" $M \otimes N$ system"), as positive semidefinite $M \times M$ block matrices, with $N \times N$ blocks (so that they are $M N \times M N$ complex matrices). (These are the elements of the unnormalized density operator, in some fixed basis of product states $e_{i} \otimes f_{j}$.) Rather than Dirac notation, we use roman letters for vectors, but we use $\dagger$ for the adjoint. Such a matrix $A$ is called separable if it can be written

$$
A=\sum_{i} x_{i} x_{i}^{\dagger} \otimes y_{i} y_{i}^{\dagger} .
$$

Objects like $x_{i} x_{j}^{\dagger}$ are outer products (in Dirac notation $\left|x_{i}\right\rangle\left\langle x_{j}\right|$; here $\left|x_{i}\right\rangle$ are not assumed normalized.). We use $e_{i}$ for elements of an orthonormal basis (typically $|i\rangle$ in Dirac notation). Thus our $e_{i} \otimes e_{j}$ would typically be written $\left|e_{i}\right\rangle \otimes\left|e_{j}\right\rangle$, or $\left|e_{i}\right\rangle\left|e_{j}\right\rangle$ or simply $|i\rangle|j\rangle$ in Dirac notation. $M_{n}$ is the set of $n \times n$ complex matrices, $M_{m n}$ the set of $m \times n$ complex matrices. When interpreting tensor products as block matrices the left-hand factor corresponds to "which block," and the right-hand to the indices within blocks.

$\|X\|$ (or $\|X\|_{\infty}$ ), with a matrix $X$ as argument, is the usual operator norm induced by Euclidean norm $\|x\|=$ $\sqrt{(x, x)}$ on vectors (i.e. $\|X\|:=\sup _{\|x\|=1}\|X x\|$ ). (It is also the $l_{\infty}$ norm of the vector of singular values of $X$, i.e. the largest singular value.) $\|X\|_{1}$ is $\operatorname{tr} \sqrt{X^{\dagger} X}$, the sum of the singular values of $X$. $\|X\|_{2}$, the Frobenius norm, is $\sqrt{\operatorname{tr} X^{\dagger} X}$, the Euclidean norm associated with the inner product $\operatorname{tr} X^{\dagger} Y$. The squared Frobenius norm is also the sum of squared singular values of $X$, and the sum of squared moduli of $X$ 's matrix elements. We write $\left[a_{i j}\right]$ for the matrix with elements $a_{i j}$.

Linear maps $\phi: M_{m} \rightarrow M_{n}$ are called positive if they preserve positive semidefiniteness. They also preseve Hermiticity (write Hermitian $H$ as a sum of positive and negative semidefinite parts, and use linearity and positivity). A stochastic map takes the identity matrix $I$ to itself. We may apply such a map $\phi$ to one subsystem of a bipartite system, while doing nothing to the other system. Applying it to the $N$-dimensional subsystem is just applying it to each block of the block matrix $X$; we call the resulting map on the bipartite system $\tilde{\phi}$ :

$$
\tilde{\phi}(X):=\left(\begin{array}{cccc}
\phi\left(X_{1,1}\right) & \phi\left(X_{1,2}\right) & \ldots & \phi\left(X_{1, N}\right) \\
\phi\left(X_{2,1}\right) & \phi\left(X_{2,2}\right) & \ldots & \phi\left(X_{2, M}\right) \\
\ldots & \ldots & \ldots & \ldots \\
\phi\left(X_{N, 1}\right) & \phi\left(X_{N, 2}\right) & \ldots & \phi\left(X_{N, N}\right)
\end{array}\right) .
$$

An important condition equivalent to separability of $A$ is that for any stochastic positive linear map $\phi, \tilde{\phi}(A)$ be positive semidefinite. We refer to it as the "Woronowicz condition." This appeared in [5], but was already essentially proven (along with the sufficiency of the partial transposition map ("Woronowicz-Peres criterion") for two qubits or a $2 \otimes 3$ system, and a $2 \otimes 4$ counterexample) in [6], though the terminology of separability and entanglement is not used there. The proof there is given for a $2 \otimes N$ system $(N<\infty)$, but it works for $M \otimes N$ by expanding the range of an index.

We also use the following fact.

Fact: Let $\Delta$ be an $M \times M$ block matrix (whose blocks need not be square). Define $\Delta^{\prime}$ as the matrix whose elements are the operator norms of the blocks of $\Delta$. Then $\|\Delta\| \leq\left\|\Delta^{\prime}\right\| \leq M\|\Delta\|$. The first inequality is wellknown; the second holds because $\left\|\Delta_{i j}\right\| \equiv\left\|P_{i} \Delta Q_{j}\right\| \leq$ $\|\Delta\| . \quad\left(P_{i}\left(Q_{i}\right)\right.$ is the projector onto the $i$-th subspace in the direct sum decomposition of the row (column) space that defines the blocks.) So, by adding to $\Delta^{\prime}$ a matrix with nonnegative entries (therefore not decreasing the norm) we can obtain $\|\Delta\|$ times the $M \times M$ all-ones matrix, whose norm is $M$.

\section{MAIN RESULT: SEPARABILITY OF PERTURBATIONS OF THE IDENTITY}

We give two proofs of the main result. Both proceed via Proposition 2, which states that stochastic positive linear maps on $n \times n$ matrices are contractive with respect to the $l_{\infty}$ ("operator") norm of matrices, for all matrices. (The result for Hermitian matrices only is much easier.) Those interested only in the shortest proof, which uses the Naimark extension, may skip to the statement of Proposition 2 below. We think it is of interest to see the connections of the norm contraction result to two different concepts well-known to quantum information theorists: in in the second proof, the Naimark extension and in the first proof, separability. The first proof proceeds via Proposition 1, which is a special case of recent results by one of us providing sufficient criteria for separability. Proposition 1: If $\|X\| \leq 1$, the block matrix

$$
\left(\begin{array}{ll}
I & X \\
X^{\dagger} & I
\end{array}\right)
$$

is separable. This is the $M=2$ case of a recent theorem (7] that all positive semidefinite $M \times M$ block Toeplitz or block Hankel matrices whose blocks are $N \times N$ matrices are separable, whose proof we include here. The paper (7] also contains two alternative proofs for the special case $M=2$. One of those proofs was independently discovered in [9].

Proof (of separability of positive semidefinite block Toeplitz matrices [7]): The proposition is a corollary to the following Lemma:

Lemma: Consider an $((M+1) \times N)$ positive semidefinite 
block Toeplitz matrix $T$ :

$$
T=\left(\begin{array}{ccccc}
R_{0} & R_{1} & R_{2} & \ldots & R_{M} \\
R_{1}^{\dagger} & R_{0} & R_{1} & \ldots & R_{M-1} \\
R_{2}^{\dagger} & R_{1}^{\dagger} & R_{0} & \ldots & \ldots \\
\ldots & \ldots & \ldots & \ldots & \ldots \\
R_{M}^{\dagger} & R_{M-1}^{\dagger} & R_{M-2}^{\dagger} & \ldots & R_{0}
\end{array}\right)
$$

(This structure is the definition of a Toeplitz matrix.) Suppose that $\operatorname{Rank}(T)=K$. Then there exist an $N \times$ $K$ matrix $X$ and a $K \times K$ unitary matrix $U$ such the $T(i, j)=X U^{i-j} X^{\dagger}, 0 \leq i, j \leq M-1$.

Proof: Since our matrix $T$ is positive semidefinite with $\operatorname{Rank}(T)=K, T=Y Y^{\dagger}$, where

$$
Y=\left(\begin{array}{lllll}
Y_{0} & Y_{1} & Y_{2} & \ldots & Y_{M}
\end{array}\right)^{T},
$$

and each block $Y_{i}$ is an $N \times K$ matrix. Define the upper submatrix $Y_{U}$ as

$$
Y_{U}=\left(\begin{array}{lllll}
Y_{0} & Y_{1} & Y_{2} & \ldots & Y_{M-1}
\end{array}\right)^{T},
$$

and, correspondingly, the lower submatrix $Y_{L}$ as

$$
Y_{L}=\left(\begin{array}{lllll}
Y_{1} & Y_{2} & Y_{3} & \ldots & Y_{M}
\end{array}\right)^{T} .
$$

It follows straight from the Toeplitz structure that $Y_{U} Y_{U}^{\dagger}=Y_{L} Y_{L}^{\dagger}$. Thus there exists an unitary $K \times K$ matrix $U$ such that $Y_{L}=Y_{U} U$ or in other words :

$$
Y=\left(\begin{array}{lllll}
Y_{0} & Y_{0} U & Y_{0} U^{2} & \ldots & Y_{0} U^{M-1}
\end{array}\right)^{T} .
$$

Recalling that $T=Y Y^{\dagger}$, we finally get the identities

$$
T(i, j)=X U^{i-j} X^{\dagger}, 0 \leq i, j \leq M-1 ; X=Y_{0} .
$$

Corollary: Using the notation of the proof above, put $U=V \operatorname{Diag}\left(z_{1}, \ldots, z_{K}\right) V^{\dagger}$ where $V$ is unitary and the complex numbers $z_{i}$ have norm one, i.e. $\overline{z_{i}}=z_{i}^{-1}, 1 \leq$ $i \leq K$. Denote the $i$ th column of $X V$ as $L_{i}$ and $Z_{i}=\left(1, z_{i}, \ldots, z_{i}^{M-1}\right)^{T}, 1 \leq i \leq K$. Then the following "separability" representation holds :

$$
T=\sum_{1 \leq i \leq K} Z_{i} Z_{i}^{\dagger} \otimes L_{i} L_{i}^{\dagger}
$$

We can use Proposition 1 to show a contraction inequality (Proposition 2 below). We got the idea of using separability to obtain operator inequalities involving stochastic positive maps from [6], where the Kadison inequality $\phi\left(X^{2}\right) \geq(\phi(X))^{2}$ for stochastic $\phi$ and hermitian $X$ is implicitly connected with the separability of:

$$
\rho=\left(\begin{array}{ll}
I & X \\
X & X^{2}
\end{array}\right) .
$$

In a sense, for $M=2$ separability of $\rho$ is equivalent to Proposition 1, as in this case there is a local unitary transformation $\rho \mapsto(A \otimes I) \rho\left(A^{\dagger} \otimes I\right)$ which maps block Toeplitz matrices to block Hankel ones (see [7] ) :

$$
A=\frac{1}{\sqrt{2}}\left(\begin{array}{ll}
1 & i \\
i & 1
\end{array}\right) .
$$

One can probably prove the next proposition using the Kadison inequality and this transformation.

Proposition 2: Let $\phi: M_{n} \rightarrow M_{n}$ be a stochastic positive linear map. Then for any $X \in M_{n},\|\phi(X)\| \leq\|X\|$.

Proof 1: We show that $\phi(X) \leq 1$ if $\|X\| \leq 1$; the proposition follows by $\phi$ 's linearity. Apply $\tilde{\phi}$ to the separable state of Proposition 1, obtaining:

$$
\left(\begin{array}{ll}
I & \phi(X) \\
\phi\left(X^{\dagger}\right) & I
\end{array}\right)
$$

Write $X=X_{1}+i X_{2}$ with $X_{1}, X_{2}$ hermitian. Then $\phi\left(X^{\dagger}\right)=\phi\left(X_{1}^{\dagger}-i X_{2}^{\dagger}\right)=$ (by Hermiticity preservation of $\phi$, which follows from its positivity) $\phi\left(X_{1}\right)^{\dagger}-i \phi\left(X_{2}\right)^{\dagger}=$ $\phi\left(X_{1}+i X_{2}\right)^{\dagger}=\phi(X)^{\dagger}$. Hence (田) is equal to:

$$
\left(\begin{array}{ll}
I & \phi(X) \\
\phi(X)^{\dagger} & I
\end{array}\right)
$$

Since this resulted from applying $\tilde{\phi}$ to a separable state, it is a positive semidefinite matrix. Positivity of this matrix is equivalent (cf. e.g. [10], p. 472) to $\phi(X)^{\dagger} \phi(X) \leq I$; i.e., $x^{\dagger} \phi(X)^{\dagger} \phi(X) x \leq 1$ for all normalized $x$, i.e. $\|X\| \leq$ 1.

This proof was independently discovered in [9]. Let us present a very different proof which does not use separability but another concept well known in the quantum information community.

Proof 2 (lifting) : It is well known that the extreme points of the matrix ball $\{X:\|X\| \leq 1\}$ are unitary matrices. Thus we can assume that $X$ is unitary, i.e. $X=\sum_{1 \leq i \leq N} z_{i} e_{i} e_{i}^{\dagger}$, where $\left|z_{i}\right|=1,1 \leq i \leq N$ and $\left\{e_{i}, 1 \leq i \leq N\right\}$ is an orthonormal basis in $C^{N}$. Thus $\phi(X)=\sum_{1 \leq i \leq N} z_{i} Q_{i}, Q_{i}=\phi\left(e_{i} e_{i}^{\dagger}\right)$. Since $\phi$ is a positive stochastic map,

$$
Q_{i} \geq 0(1 \leq i \leq N) \text { and } I=\sum_{1 \leq i \leq N} Q_{i} .
$$

By Naimark's theorem 11] [12] (cf. 13] for a simple exposition in finite dimension) there exist commuting orthogonal projectors $P_{i}: C^{K} \rightarrow C^{K}, N \leq K \leq N^{2}$, and a unitary injection $U: C^{N} \rightarrow C^{K}$ such that

$$
Q_{i}=U^{\dagger} P_{i} U(1 \leq i \leq N) \text { and } I=\sum_{1 \leq i \leq N} P_{i} .
$$

It is easy to see that $\left\|\sum_{1 \leq i \leq N} z_{i} P_{i}\right\| \leq 1$. Thus

$$
\left\|\sum_{1 \leq i \leq N} z_{i} Q_{i}\right\| \leq\left\|U^{\dagger} \mid\right\|\left\|\sum_{1 \leq i \leq N} z_{i} P_{i}\right\|\|U\| \leq 1 .
$$

This second proof suggests that there might be a deeper connection between Naimark's theorem and separability. 
We proceed to the main theorems.

Theorem 1: The matrix $I+\Delta$ is separable for all hermitian $\Delta$ with $\|\Delta\|_{2} \leq 1$.

Proof:

$$
\|\tilde{\phi}(\Delta)\|^{2} \leq\|A\|^{2} \leq\|A\|_{2}^{2},
$$

where $A:=\left[a_{i j}\right], a_{i j}:=\left\|\phi\left(\Delta_{i j}\right)\right\|$.

$$
\|A\|_{2}^{2}=\sum_{i j} a_{i j}^{2}=\sum_{i j}\left\|\phi\left(\Delta_{i j}\right)\right\|^{2} .
$$

(The first inequality is because the operator norm of a block matrix is bounded above by that of the matrix whose elements are the norms of the blocks, and the second is because the Frobenius norm is an upper bound to the operator norm.) But $\left\|\phi\left(\Delta_{i j}\right)\right\|^{2} \leq\left\|\Delta_{i j}\right\|$ by Prop. 2 , and this in turn is less than $\left\|\Delta_{i j}\right\|_{2}$. So

$$
\|\tilde{\phi}(\Delta)\|^{2} \leq \sum_{i j}\left\|\phi\left(\Delta_{i j}\right)\right\|^{2} \leq \sum_{i j}\left\|\Delta_{i j}\right\|_{2}^{2} \equiv\|\Delta\|_{2}^{2} \leq 1,(9)
$$

the last inequality being the premise of the theorem. Having shown that $\|\tilde{\phi}(\Delta)\| \leq I$, and also using $\tilde{\phi}(I)=I$, we get $\tilde{\phi}(I+\Delta) \geq 0$, so that by Woronowicz' criterion $I+\Delta$ is separable.

\section{COROLLARIES AND ADDITIONAL RESULTS: MAXIMALITY OF BALLS, SCALING AND SPECIFIC PERTURBATIONS}

Let us now present some corollaries of Theorem 1. Define $\|\Delta\|_{p}:=\left(\sum_{i}\left|\lambda_{i}\right|^{p}\right)^{\frac{1}{p}}\left(\lambda_{i}\right.$ being the eigenvalues of the square hermitian matrix $\Delta$.)

Corollary 1 ( $l_{p}$ balls): Consider an $N \times N$ system. Then the matrix $I+\Delta$ is separable for all hermitian $\Delta$ with $\|\Delta\|_{p} \leq 1(1 \leq p \leq 2)$ and $\|\Delta\|_{p} \leq B(N, p)=: N^{\frac{2}{p}-1}(2 \leq$ $p \leq \infty)$.

Proof: The statements follow from basic $p$-norm inequalities: the first from the $q=2$ cases of $\|\Delta\|_{p} \geq\|\Delta\|_{q}$ (for $1 \leq p \leq q$ ), the second from the $q=2$ cases of $\|\Delta\|_{q} \leq n^{\frac{1}{q}-\frac{1}{p}}\|\Delta\|_{p}$ (for $p \geq q$ ). Note that the dimension $n$ is $N^{2}$ in our case. (These inequalities are equivalent to similar ones for the vector $p$-norms $\|x\|_{p}:=\left(\sum_{i} x_{i}^{p}\right)^{\frac{1}{p}}$; the first set can be proved by changing variables to $y_{i}=x_{i}^{p}$ and using the triangle inequality for norms, the second by letting $y_{i}=x_{i}^{q}$ and using the convexity of $f: z \mapsto z^{\alpha}$ for $\alpha \geq 1$ ( $\alpha=p / q$ in our case).)

The $l_{\infty}$ result was also obtained very recently in [9] using quite different methods. The $p$-balls in Cor. 1 are clearly the largest possible for $1 \leq p \leq 2$ : by subtracting any normalized pure state, for which all these $p$-norms are 1 , we can leave the positive, and hence the separable, cone. What about $2<p \leq \infty$ ? Theorem 3 will show that these are the largest balls for these norms, too.

The next theorem gives information about how fast we can reach the entangled states by perturbing the identity in a specific direction: adding a positive multiple of a pure state. The main point is that, perhaps surprisingly, the entangled states are reached fastest by perturbing with a $2 \otimes 2$ Bell state, rather than, say, a maximally entangled state.

Theorem 2 (Perturbation by positive multiples of pure states): 1 . Consider a pure $\rho$ corresponding to a state $\left.|\psi\rangle=\sum_{i j} \psi_{i j} e_{i} \otimes e_{j}, 1 \leq i, j \leq N\right\}$. The the spectrum of $\rho^{T}$ is $\left(d_{1}^{2}, \ldots, d_{N}^{2} ; d_{i} d_{j},-d_{i} d_{j}(1 \leq i \neq j \leq N)\right)$, where $d_{1}, \ldots, d_{N}$ are the singular values of the $N \times N$ matrix $\psi:=\left[\psi_{i j}: 1 \leq i, j \leq N\right]$ (thus $\left.d_{1}^{2}+\ldots+d_{N}^{2}=1\right)$.

2. Define

$$
W(N)=\min _{\rho \in \operatorname{Den}(N, N)}-\lambda_{\min }\left(\rho^{T}\right),
$$

where $\operatorname{Den}(N, N)$ is the set of density matrices of $N \times N$ systems. Then $W(N)=\frac{1}{2}$.

3. If $I+a \rho$ is separable for all $\rho$ and $a>0$ then $a \leq W(N)^{-1}=2$.

Proof: Diagonalize $\psi$ by local unitaries using the singular value ("Schmidt") decomposition obtaining $\operatorname{Diag}\left(d_{1}, \ldots, d_{N}\right)$. The corresponding density matrix has blocks $\rho_{i j}=d_{i} d_{j} e_{i} e_{j}^{\dagger}$, and the spectrum of $\rho^{T}$ (which is not changed by applying local unitaries prior to partial transposition) is as given in Part 1 of Thm.2. The bound in Part 2 follows from $2 a b \leq a^{2}+b^{2}$ and is achieved by $(1 / \sqrt{2}, 1 / \sqrt{2}, 0, \ldots, 0)$. The Woronowicz-Peres (WP) condition gives Part 3.

Contrary to the "folklore," in the result above the fully entangled state is not the worst one; rather, the worst is a maximally entangled state of two local two-dimensional subspaces.

Theorem 3 establishes the maximum size of the $p$-balls for $p>2$. The proof involves considering perturbations by a positive multiple of the maximally entangled state and establishes when this procedure hits the entangled states. Before formulating the theorem we introduce some notation. $W P(N, N)$ is the closed convex cone of $N^{2} \times N^{2}$ positive matrices satisfying the WP condition, i.e. $\rho \in W P(N, N)$ iff $\rho \geq 0$ and $\rho^{T} \geq 0 . \operatorname{Sep}(N, N)$ is the closed convex cone of separable positive matrices. Obviously $W P(N, N) \subset \operatorname{Sep}(N, N)$. Both $W P(N, N)$ and $\operatorname{Sep}(N, N)$ are subsets of the real $N^{4}$-dimensional linear space $H\left(N^{2}\right)$ of hermitian $N^{2} \times N^{2}$ matrices. The cone dual to a convex set $X$ (which need not be a cone) is $X^{*}:=\{y:\langle y, x\rangle \geq 0, \forall x \in X\}$.

Theorem 3: Suppose $p>2$. If the $p$-ball $\operatorname{Ball}(N, p, a)=$ $\left\{A \in H\left(N^{2}\right): A=I+\Delta,\|\Delta\|_{p} \leq a\right\}$ belongs to $W P(N, N)$ then $a \leq B(N, p)=: N^{-1+\frac{2}{p}}(2 \leq p \leq \infty)$.

As $W P(N, N) \subset \operatorname{Sep}(N, N)$ this Theorem proves that the $l_{p}$-balls $\operatorname{Ball}(N, p, B(N, p))$ in Corollary 1 are largest possible.

Proof of Theorem 3: It is easy to see that the cone dual to $\operatorname{Ball}(N, p, a)$, i.e. $\operatorname{Ball}(N, p, a)^{*}$, is $\{A \in$ $\left.H\left(N^{2}\right): \operatorname{tr}(A) \geq a\|A\|_{q}, q=\frac{p}{p-1}\right\}$. It is known [6] that $W P(N, N)^{*}=\left\{\rho_{1}+\rho_{2}^{T}: \rho_{i} \geq 0, i=1,2\right\}$. If $\operatorname{Ball}(N, p, a) \subset W P(N, N)$ then $W P(N, N)^{*} \subset$ 
$\operatorname{Ball}(N, p, a)^{*}$ and at least $\operatorname{tr}(\rho)=\operatorname{tr}\left(\rho^{T}\right) \geq a\left\|\rho^{T}\right\|_{q}$ for all $\rho \geq 0$. Consider the fully entangled pure $N \times N$ state $\rho_{E}$. Then $\operatorname{tr}\left(\rho_{E}\right)=1$. It follows from Part 1 of Thm. 2 that $\rho_{E}^{T}$ has $N+\frac{N(N-1)}{2}$ eigenvalues equal to $\frac{1}{N}$ and $\frac{N(N-1)}{2}$ eigenvalues equal to $\frac{-1}{N}$. Thus we get that $1 \geq a\left\|\rho_{E}\right\|_{q}=a N^{\frac{2-q}{q}}$ and, finally, $a \leq N^{\frac{q-2}{q}}=N^{\frac{2}{p}-1}$.

The following corollary of Theorem 1 gives (as is evident from the proof) the strongest sufficient condition for separability of $A \geq 0$ that can be derived by scaling (considering all ways of writing $A=\zeta(I+\Delta)$ with $\zeta>0$ ) and using the Frobenius norm case of Theorem 1 (applied to $\Delta)$.

Corollary 2 (scaling) : Let $A$ be an (unnormalized) density matrix of a bipartite system with total dimension $d=N M$ and $\lambda=\left(\lambda_{1}, \ldots, \lambda_{d}\right)$ be the vector of eigenvalues of $A$. If

$$
S(\lambda)=: d-\frac{\|\lambda\|_{1}^{2}}{\|\lambda\|_{2}^{2}} \leq 1
$$

then $A$ is separable.

Proof: It is easy to see that $S(\lambda)=\min _{a>0}\|a \lambda-e\|_{2}^{2}$, where $e$ is a vector of all ones. Therefore if $S(\lambda) \leq 1$ then $A=b(I+\Delta)$, where $b>0$ and $\|\Delta\|_{2}^{2} \leq 1$. It follows from Theorem 1 that $A=b(I+\Delta)$ is separable.

Corollary 3 (largest Frobenius ball for density matrices): Suppose that $A$ is a normalized density matrix of a bipartite system with total dimension $d=N M$, i.e. $\quad \sum_{1 \leq i \leq d} \lambda_{i}=1$ and $\lambda_{i} \geq 0,1 \leq i \leq d$. If $\left\|A-\frac{1}{d} I\right\|_{2}^{2}=\left\|\lambda-\frac{1}{d} e\right\|_{2}^{2} \leq \frac{1}{d(d-1)}=: r^{2}$ then $A$ is separable. $r$ is the largest such constant.

Proof: Define $t=\lambda-\frac{1}{d} e$. Then $\|\lambda\|_{1}^{2}=1$ and $\|\lambda\|_{2}^{2}=$ $\frac{1}{d}+\|t\|_{2}^{2}$. Thus $S(\lambda)=d-\frac{1}{\frac{1}{d}+\|t\|_{2}^{2}}$ and $S(\lambda) \leq 1$ iff $\|t\|_{2}^{2} \leq$ $\frac{1}{d(d-1)}$. From Corollary 2 it follows that $A$ is separable. On the other hand $r=1 / \sqrt{d(d-1)}$ is the radius of the largest ball inside the $d$-dimensional simplex.

Remark: In terms of the "purity" $\operatorname{tr} \rho^{2}$ of the density matrix (which takes the value 1 for pure states and $1 / d$ for the maximally mixed state), Corollary 3 says that $\rho$ is separable if its purity is less than or equal to $1 /(d-1)$.

One might conjecture that for $N \times N$ bipartite systems ( so $d=N^{2}$ ), any $\lambda$ not satisfying $(10)$ is the spectrum of some non-separable positive matrix. This is not so: a sufficient condition for separability of two-qubit density matrices in terms of the spectrum is [14] $\lambda_{1}-\lambda_{3}-\sqrt{\lambda_{2} \lambda_{4}} \leq 0$, where $\lambda_{i}$ are decreasingly ordered. This can hold when the purity is greater than $1 / 3$, as also noted in [15].

Corollary 4: The matrix $I+a \rho$, where $\rho$ is an $N \times N$ state, is separable if $-1 \leq a \leq \frac{N^{2}}{N^{2}-2}$.

Proof: Clearly it is enough to prove this for pure states. In this case the vector of eigenvalues of $I+a \rho$ is

$$
\lambda_{a}=:(1,1, \ldots, 1,1+a) .
$$

Direct computation gives that $S\left(\lambda_{a}\right)=N^{2}-\frac{\left(N^{2}+a\right)^{2}}{N^{2}+2 a+a^{2}}$. It follows that $S\left(\lambda_{a}\right) \leq 1$ iff $-1 \leq a \leq \frac{N^{2}}{N^{2}-2}$.
Corollary 5: If we consider the normalized mixtures $\sigma=$ $(1-\epsilon) I / d+\epsilon \rho$, for pure $\rho$, and scale them as

$$
\sigma=\frac{1-\epsilon}{d}\left(I+\frac{d \epsilon}{1-\epsilon} \rho\right),
$$

by Corollary 4 these are separable if $\epsilon \leq 1 /(d-1) \equiv$ $1 /\left(N^{2}-1\right)$.

A very slightly better, but messier, bound can be obtained by solving a quadratic equation derived from Cor. 2 , reminding us that the most obvious or tractable scaling is not generally the best. Corollary 5 is of course also true for mixed $\rho$.

\section{PERTURBATION OF THE IDENTITY BY POSITIVE MULTIPLES OF PROJECTORS}

A final result again illustrates the power of scaling (Corollary 2). It gives us the most negative eigenvalue of a partial transpose of a projector on a bipartite system. This is interesting because it tells us when we will hit the entangled matrices if we add a positive multiple of that projector to the identity. Define

$$
W_{m}(N):=\min _{\rho \in P R(m, N)}-\lambda_{\min }\left(\rho^{T}\right)
$$

where $P R(m, N)$ stands for the compact set of all rank $m$ orthogonal projectors in $C^{N} \otimes C^{N}$. Notice that part 2 of Theorem 2 states that $W_{1}(N)=W(N)=\frac{1}{2}$; clearly $W_{N}(N)=0$, it follows from Theorem 1 that also $W_{N-1}(N)=0$; it is easy to prove that if $\frac{K}{L}$ is an integer then $\frac{W_{K}(N)}{K} \leq \frac{W_{L}(N)}{L}$.

Theorem 4:

$$
W_{\frac{N(N-1)}{2}}(N)=\frac{N-1}{2} .
$$

Proof: Let us define the following operator intervals :

$$
\operatorname{Int}(a, N)=:\left\{\rho: C^{N} \otimes C^{N} \rightarrow C^{N} \otimes C^{N}:(1+a) I \geq \rho \geq I\right\}
$$

It follows by a straightforward rescaling from the $l_{\infty}$ part of Corollary 1 and Theorem 3 that

Property 1 if $0 \leq a \leq \frac{2}{N-1}$ then all matrices in $\operatorname{Int}(a, N)$ are separable (and thus satisfy the Woronowicz-Peres condition) .

Property 2 If $a>\frac{2}{N-1}$ then there exists a matrix in $\operatorname{Int}(a, N)$ which does not satisfy the WoronowiczPeres condition.

It is easy to see that the extreme points of the compact convex set $\operatorname{Int}(a, N)$ are of the form $I+a P$, where $P$ is an arbitrary orthogonal projector; correspondingly $d$ dimensional vectors composed of eigenvalues of extreme points have (up to permutations) the following form:

$$
\lambda_{m, a}=e+a V_{m}, 0 \leq m \leq d=N^{2},
$$


where $e$ is the all-ones vector and vector $V_{m}$ has its first $m$ coordinates equal to one and the rest equal to zero. Simple algebra gives that $S\left(\lambda_{\frac{N(N-1)}{2}, \frac{2}{N-1}}\right)=1$ and $S\left(\lambda_{k, \frac{2}{N-1}}\right)<1$ for all $k \neq \frac{N(N-1)}{2}$. Therefore if $\epsilon>0$ is small enough then $S\left(\lambda_{k, \frac{2}{N-1}+\epsilon}\right)<1$ for all $k \neq \frac{N(N-1)}{2}$. Corollary 2 implies that for all small enough $\epsilon>0$ matrices $I+\left(\frac{2}{N-1}+\epsilon\right) P$ are are separable (and thus satisfy the Woronowicz-Peres condition) provided that $P$ is an orthogonal projector of rank $k \neq \frac{N(N-1)}{2}$. It follows from Property 2 above that for all $a>\frac{2}{N-1}$ there exists an orthogonal projector $P_{a}$ such that $I+a P_{a}$ does not satisfy the Woronowicz - Peres condition, in other words that

$$
\left|\lambda_{\min }\left(P_{a}^{T}\right)\right|>a^{-1} .
$$

It follows that if $a=\frac{2}{N-1}+\epsilon$ and $\epsilon>0$ is small enough then necessarily

$$
\operatorname{Rank}\left(P_{a}\right)=\frac{N(N-1)}{2}
$$

Thus $W_{\frac{N(N-1)}{2}}(N) \geq \frac{N-1}{2}$, but Property 1 above implies that $W_{\frac{N(N-1)}{2}}(N) \leq \frac{N-1}{2}$. Therefore

$$
W_{\frac{N(N-1)}{2}}(N)=\frac{N-1}{2} .
$$

One implication of this result is that the boundary of the largest $p=2$ ball also contains points in the interior of the postive cone. One sees this by noting that $a=2 /(N-1)$ is the largest $a$ for which $I+a P$ is separable, where $P$ is the rank- $N(N-1) / 2$ projector achieving the value $W_{N(N-1) / 2}=(N-1) / 2$ of Theorem 4 . For all greater $a$, the matrix is entangled; so is the scaled operator $(N-1) / N$ times this matrix. But for $a=2 /(N-1)$ this scaled matrix satisfies $((N-1) / N)(I+a P)=I+\Delta$, where $\Delta$ is Hermitian with $N(N+1) / 2$ eigenvalues $-1 / N$ and $N(N-1) / 2$ eigenvalues $+1 / N$. This is well within the interior of the positive cone, and $\|\Delta\|_{2}=1$.

\section{DISCUSSION AND CONCLUSION}

For a product of $R N$-dimensional systems in a mixture

$$
\rho=(1-\epsilon) I / N^{R}+\epsilon \rho^{\prime},
$$

( $\rho^{\prime}$ a normalized density matrix), [ [4, extending [3], found lower and upper bounds on the value $\epsilon_{\max }(\rho)$ below (and at) which the state can be guaranteed to be separable: $1 /\left(1+N^{2 R-1}\right) \leq \epsilon_{\max }(\rho)<1 /\left(1+N^{R-1}\right)$. (The $N=$ 2 case is in [3].) For bipartite systems $(R=2)$, these bounds are $1 /\left(1+N^{3}\right)$ and $1 /(1+N)$. The lower bound is close to what one can get from the $l_{\infty}$ (operator norm) result, while the upper bound comes from mixing in the maximally entangled $\rho_{E}$. The results of this paper give $1 /\left(N^{2}-1\right) \leq \epsilon_{\max }(\rho) \leq 2 /\left(2+N^{2}\right)$. The lower bound is via Corollary 5 of Theorem 1 , and is tighter due to the use of Frobenius rather than operator norm; the upper bound uses Theorem 2 and the same scaling as in Cor. 5 , and is tighter because the maximally entangled state is not the optimal state to mix in.

Our knowledge of the exact size of the 2-norm ball in the bipartite case, gives us a bound exponentially better than known bounds on $\epsilon_{\max }(\rho)$. This shows how much more powerful our sufficient condition for separability is than previously known geometric conditions, in the bipartite setting.

It is also illuminating to investigate the implications of our results in the multipartite setting; we will com-

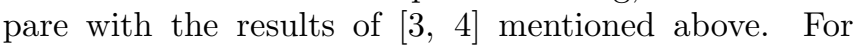
multipartite states $(R>2)$ we get a slightly better upper bound on $\epsilon_{\max }(\rho)$. For example, for even $R$, $\epsilon_{\max }(\rho) \leq 2 /\left(2+N^{R}\right)$, by dividing the systems into equal sized sets and viewing the state as bipartite. For qubits, this is actually the same as in [3], although since we used a slightly less than optimal scaling, we could improve it a bit. Our results also imply that no matter what state $\rho$ is mixed in, if $\epsilon \leq 1 /\left(N^{R}-1\right)$, the state is separable with respect to every bipartition. This is dramatically larger than [3]'s bound below which the state is guaranteed separable, but not directly comparable because a state can be separable with respect to every such bipartition yet not be separable 16. This raises the important question of the size of the largest separable ball in the multipartite case, to which we expect our methods can contribute.

In conclusion, we have found the exact size of the largest $p$-norm balls of entangled states around the identity, for all $1 \leq p \leq \infty$, and established for $p=2$ that the edge of the ball can be reached within the positive cone. Applied via scaling as we illustrated with several corollaries and examples, this yields sufficient conditions for separability which can be exponentially stronger in many situations than previously known conditions. In particular, we found the strongest such condition statable in terms of the spectrum of a density matrix, and derivable via scaling of the $p=2$ result: for normalized density matrices of $d \otimes d$ systems, it is that the purity $\operatorname{tr} \rho^{2}$ be less than $1 /(d-1)$. In addition, for three special classes of perturbations (positive multiples of pure states, positive multiples of the maximally entangled state, and positive multiples of projectors), we found the smallest perturbation in the class achieving entanglement. The pure state result, that it is a $2 \otimes 2$ Bell state rather than an $N \otimes N$ maximally entangled state, is not only mathematically interesting but transparent in meaning, and possibly surprising. These are natural special classes of perturbations that have been previously considered in quantum information theory, so we expect that these results will find application in many appropriate situations. Because of the natural geometric form of our general sufficient conditions for separability (Theorem 1) and related results, their status as a basic aspect of the geometry of the entangled states, and the important role of these balls in computational questions, we anticipate many applications for them, in theory and in the interpretation 
and engineering of experiments that aim to produce entanglement.
Thanks to Manny Knill for discussions, and the US DOE and NSA for support.
[1] R. Jozsa and N. Linden (2002), arXiv.org preprint quant$\mathrm{ph} / 0201143$.

[2] K. Zyczkowski, P. Horodecki, A. Sanpera, and M. Lewenstein, Phys Rev A 58, 883 (1998).

[3] S. Braunstein, C. M. Caves, R. Jozsa, N. Linden, S. Popescu, and R. Schack, Phys Rev Lett 83, 1054 (1999).

[4] P. Rungta, W. J. Munro, K. Nemoto, P. Deuar, G. J. Milburn, and C. M. Caves (2001), in Directions in Quantum Optics: A Collection of Papers Dedicated to the Memory of Dan Walls, D. Walls, R. Glauber, M. Scully, and H. Carmichael, eds. Springer, 2001; also arXiv.org preprint quant-ph/0001075.

[5] M. Horodecki, P. Horodecki, and R. Horodecki, Phys Lett A 223, 1 (1996).

[6] S. L. Woronowicz, Rep Math Phys 10, 165 (1976).

[7] L. Gurvits (2001), LANL Unclassified Technical Report LAUR-01-2030.
[8] L. Gurvits (2002), ArXiV.org e-print quant-ph/0201022.

[9] T. Ando (2001), preprint, Hokusei Gakuen University.

[10] R. A. Horn and C. R. Johnson, Matrix Analysis (Cambridge University Press, Cambridge, 1985).

[11] M. A. Neumark, Izv. Akad. Nauk. SSSR, Ser. Mat. 4 53, 277 (1940).

[12] M. A. Neumark, C. R. (Doklady) Acad. Sci. URSS (N.S.) 41, 359 (1943).

[13] A. Peres, Quantum Theory: Concepts and Methods (Kluwer Academic, Dordrecht, 1993).

[14] F. Verstraete, K. Audenaert, and B. De Moor, Phys Rev A 64, 012316 (2001).

[15] K. Zyczkowski and M. Kús, Phys Rev A 63, 032307 (2001).

[16] C. H. Bennett, D. P. DiVincenzo, T. Mor, P. W. Shor, J. A. Smolin, and B. M. Terhal, Phys Rev Lett 82, 5385 (1999). 